\title{
(2) OPEN ACCESS \\ Creating an ethical culture to support recovery from substance use disorders
}

\author{
Laura Williamson
}

Center for Bioethics and Health Policy, Institute of Public \& Preventive Health, Augusta University, Augusta, Georgia, USA

\section{Correspondence to}

Dr Laura Williamson, Center for Bioethics and Health Policy, Institute of Public \& Preventive Health, Augusta University, Augusta, GA 30912, USA; LAURAWILLIAMSON07@ GMAIL.COM

Received 2 July 2020

Revised 7 September 2020 Accepted 23 September 2020

Check for updates

(C) Author(s) (or their employer(s)) 2020. Re-use permitted under CC BY-NC. No commercial re-use. See rights and permissions. Published by BMJ.

\begin{tabular}{|l|}
\hline To cite: Williamson L. \\
J Med Ethics Epub ahead of \\
print: [please include Day \\
Month Year]. doi:10.1136/ \\
medethics-2020-106661 \\
\hline
\end{tabular}

\section{ABSTRACT}

There is a long-standing failure to create an ethical culture around substance use disorders (SUDs) or dependence that actively supports people's recovery efforts. Issues which impede the development of prorecovery environments are complex, but include the far-reaching effects of the social stigma that surrounds SUDs; and the failure to harness relational and social support that allows debates to transcend blaming individual substance users. As part of efforts to create prorecovery environments, it is important to acknowledge that bioethics debate on SUDs is narrow in scope, prioritising topics related to its traditional interests in individual autonomy and novel technologies. As a result, it has not played a significant role in helping to transform the ethical cultures in which substance use recovery takes place. For example, it largely neglects the ethical challenges of developing an empathic, person-centred approach to substance use problems that listens and responds to the voices of clients. It has also participated little in efforts to develop a positive response to reducing the toxic effects of stigma. Indeed, some contributions from the field fan stigma, rather than alleviate it. The aim of this paper is to seed broader ethical debate, in academic literature and lay/professional communities, on how societies should respond to SUDs: steering a course between the critical, but narrow approach of bioethics and the empowerment discourse of evidence-based treatments.

\section{INTRODUCTION}

Substance use disorders (SUDs) or dependence ${ }^{12}$ are responsible for substantial health, economic and social costs. ${ }^{3}$ The opioid crisis, ${ }^{4}$ and a sharp rise (49.4\%) in alcohol use disorders in the USA, ${ }^{5}$ evidence the importance of urgently addressing such conditions. Providing better treatment and prevention for SUDs is part of the United Nation's Sustainable Development Goals. ${ }^{6}$ An array of behavioural and pharmacological interventions exist for substance use problems with a growing evidence base to support their use. ${ }^{78}$ For example, in addition to familiar 12-step support, social skills training, motivational aid and community reinforcement are also available. ${ }^{9}{ }^{10}$ But the treatment people access, for a variety of reasons, can be substandard. ${ }^{11}$ Furthermore, research showing people recover from SUDs without treatment, makes it particularly important to understand the role of a person-centred approach in this context. ${ }^{12}$ Finally, other routes to controlling SUDs like, harm reduction strategies, require more attention even though they are often politically unpopular. ${ }^{13}$

Barriers to quality treatment uptake and the recovery process are complex, but rooted in a toxic interplay of social and clinical factors, including: cost, lack of availability, consultation style and people being too ashamed to seek help due to SUDs being heavily stigmatised. ${ }^{11}$ The actual treatment people receive is only part of efforts to overcome SUDs. Also important are the environments in which recovery takes place, including the individual and social values that inform treatment provision and recovery contexts.

In the USA, the Substance Abuse and Mental Health Service and Administration (SAMHSA) defines recovery as a process 'through which individuals improve their health and wellness, live a self-directed life and strive to reach their full potential'. ${ }^{14}$ It continues to articulate that this process requires 'individuals to optimise their autonomy and independence' by controlling their health-related choices; and social or relational aid that provides 'hope, support and encouragement' including 'strategies and resources for change'. ${ }^{14}$ The reliance on both the autonomy and independence of individuals, and supportive relationships for recovery raises questions over how these two perspectivesindividual choice and social support-can coexist in the context of SUDs. This is not least because the choices of people with SUDs are often branded as 'irresponsible' and, partly as a result, stigmatised. For the individual and social elements of the recovery process to work positively together it is important to afford them greater ethical attention.

I begin by briefly examining the contribution of bioethics to SUDs. I will then consider two areas that require ethical support to help make social and clinical environments prorecovery, namely: empathic, person-centred care (PCC) and efforts to use affirmation to overcome the negative impacts of stigma. The aim of the paper is to seed greater consideration, in academic literature and lay/ professional communities, of fundamental ethical issues that undermine cultivating an ethical culture that supports, rather than thwarts people's recovery efforts.

\section{BIOETHICS AND ADDICTION}

Ethical tensions associated with SUDs have received much attention in bioethics and in wider philosophical literature. Despite the volume of bioethics material that exists, the discussions it presents tend to be quite limited in scope. In this respect, bioethics debate coalesces around themes that are influenced by its traditional interest areas: the nature and protection of individual autonomy, rationality and related issues like informed consent ${ }^{15}$; and the implications of developing and implementing novel technologies. ${ }^{16}$ This has led to debates on issues 
such as: whether people with SUDs should be held morally responsible for their actions ${ }^{17}$; the ability of people with SUDs issues to give informed consent to participate in treatment or research $^{18}$; the acceptability of coercing people into treatment ${ }^{19}$; the implications for individual freedoms of drug screening and genetic testing ${ }^{20}$ and the ethical implications of novel drug treatments, including vaccinations against illicit drug use. ${ }^{21}$

Despite its focus on autonomy, it is curious that bioethics has seldom entered the clinic's doors and into the experience of people living with SUDs, or the social challenges presented. As Silkoff et al note, there is also is a failure to ethically support healthcare practitioners in the field. Their review shows that the dominance of rule or principle-based guidance for SUDs rarely considers the value of relationships in recovery and the ethical challenges of supporting them. ${ }^{22}$ While there are some exceptions to this characterisation, ${ }^{2324}$ the footprint of bioethics in the context of SUDs is not one that helps to create a positive prorecovery environment. Its focus is too often blind to the importance of finding ways to cultivate humanising, person-centred responses to SUDs that acknowledge the individual and social challenges of recovery. I will now advance to examine themes that evidence-based research in the field highlights as important: relational empathic, PCC and positive ways of eradicating or minimising stigma.

\section{PCC AND SUPPORTIVE, EMPATHIC RELATIONSHIPS}

Commitments to PCC are well established in health systems. A person-centred approach is driven by practical and ethical imperatives to elicit the values and preferences of service users. ${ }^{25}$ Among the practical drivers of PCC are the need to provide quality care that is tailored to the service users' particular needs and preferences in an efficient and sustainable manner. Ethical drivers for PCC include the need to safeguard the values and rights of individuals. ${ }^{26}$ It is this same type of person-centred approach which features in the recovery process outlined by SAMHSA. ${ }^{14}$ The Institute of Medicine has also recommended that, despite concerns over intermittently impaired decision making being associated with SUDs, PCC should and can be used in this field. ${ }^{11}$

Related to PCC is literature supporting the importance of effective therapeutic relationships in the context of SUD treatment. A strong therapeutic alliance is as significant as the type of treatment a person receives. ${ }^{27}$ As Miller et al note 'treatment method is not the only, and often not even the primary determinant of client outcomes. ${ }^{9}$ The variation in the success of therapists, including some with particularly poor outcomes, ${ }^{28}$ raises concerns over the performance of individual counsellors, therapists and treatment facilities. Research suggests there are a range of relational factors that have a positive impact, including: therapists having 'strong interpersonal skills' ${ }^{28}$ alliance building with clients, collecting client feedback and empathy. ${ }^{27}$ Empathic consultations with clients have been presented as a significant influence on achieving positive outcomes. Rogers describes empathy as 'temporarily living in the other's life, moving about in it delicately without making judgements'. ${ }^{29}$ The importance of strong, empathic relationships with clients has been linked to their ability to assist people to feel 'safe and understood', ${ }^{29}$ thereby helping to overcome their sense of alienation, resistance to assistance and behavioural change. Conversely, confrontational consultation styles can result in more resistance from clients. $^{3031}$

Despite commitments to PCC, the treatment people with SUDs receive can fail to deliver the type of care that respects people's choices and values. Indeed, it has been argued that the care provided to those with SU problems stands in 'stark contrast' to the requirements of a person-centred approach. ${ }^{32}$ For example, people are offered a restricted range of treatment options based around a 12-step model and, as a result, limited pharmacological support ${ }^{32}$; and healthcare professionals can hold negative views of SUDs, which impacts on the treatment they provide. ${ }^{11}$ The problems dogging SU treatment provision are exemplified by a case in which a nurse in Canada recently won a legal challenge against his employer-a healthcare institution-for mandating participation in Alcoholics Anonymous, and terminating his position when he did not attend. As an atheist, he did not want to participate in a programme with a spiritual dimension..$^{33}$ As other evidence-based treatments exist, ignoring a person's choice in this way fails to respect commitments to PCC; and suggests that people with SUDs can be treated less favourably than those with other health conditions. As White has argued, doubts around the capacity of people with SUDs to make choices about their treatment need, in the context of PCC and diverse recovery options, to be replaced by increasing the role people have in their own recovery. ${ }^{34}$ It is, therefore, important to give greater ethical attention to failures to provide PCC in the context of SUD treatment to help strengthen its role in creating a prorecovery ethical culture.

\section{Person-centred care: more than less paternal}

A person-centred approach is often grounded in a traditional account of autonomy linked to self-determination and independence. ${ }^{35}{ }^{36}$ We have seen such a reading of autonomy is also linked to recovery. However, this foundation is too individually focused to help develop an ethical culture that easily supports recovery. There are a number of reasons for this: a simple focus on self-determination, while intended to empower, does not provide sufficient support to help us move beyond seeing people as solely responsible for what is a complex biopsychosocial condition. The focus remains on the person seeking treatment, not the professionals providing it. It also gives inadequate decision-making support to help people work through what is the best course of action for them: independence has been linked to abandonment. ${ }^{37}$ Finally, a focus on self-determination lacks the ability to highlight that what a person-centred approach requires is not leaving people to their own devices (by being less paternalistic), but actively engaging with and supporting them to make informed choices about their recovery options.

In the context of SUDs, the importance of PCC being more than less paternalistic are, for example, illustrated by Schwartz et $a l^{38}$ in their study that questions the value of PCC in the context of methadone treatment. The study understands PCC as providing treatment 'tailored to the patients' preferences and needs'. It aims to achieve this by participants being 'encouraged but not required to attend individual and/or group counselling'; and participants not being 'discharged for rule infractions' such as not attending counselling, failing to pay fees and loitering. ${ }^{38}$ Measuring such features, however, does not meaningfully assess a person-centred approach to substance use. This is because making treatment 'less disciplinarian' does not necessarily capture the preferences of clients; rather it paternalistically imposes what the authors assume clients might want. The only way to determine this is to ask clients about their priorities and choices. It is feasible that some clients may prefer the order and regime that the study authors consider contrary to PCC and autonomy. A person-centred approach that does not engage with a person's values or support their choices, is person centred in name only. 
In the context of SUDs, not engaging with the views of service users could point, not only to an implementation failure, but a deep antipathy to valuing the preferences or choices of people in this stigmatised group. Given this, it is important to examine strategies for increasing the attention afforded to the perspective of people with SUDs as part of efforts to improve the ethical culture in which recovery takes place.

\section{Empathy and substance use disorders}

As noted above, therapist empathy is thought to play a significant role in psychosocial therapies as a way to overcome client alienation and to help their engagement with treatment. However, a ramification of the lack of ethical attention given to relationships in recovery, is that empathy is also underexamined. Yet beyond the substance use field, there is much ethical debate on this topic which helps to highlight some of the challenges surrounding the use of empathy to support recovery.

Ethical thought on empathy highlights the lack of clarity over what it requires and the need for greater specificity. ${ }^{39}$ While the nature of empathy is disputed, there is agreement it should not be reduced to mimicking the experience of others, emotional contagion, nor be confused with sympathy. ${ }^{3940}$ The key to empathising is getting 'inside' another person's 'situated psychological states while maintaining clear self-other differentiation'. ${ }^{39}$ This raises questions over whether it is possible to accurately know other peoples' experience. van Dijke et $a l^{41}$ note that empathy has been rejected as a form of projection that does not really help us understand the experience of others. However, even if we do not reject the possibility of empathising with others, ethical questions remain. There are, for example, concerns that empathy might result in poor or unethical decisions. ${ }^{42}$ While valuable, reliance on empathy could, therefore, yield too much control to poor judgements and too little to corrective, professional guidance. Also for debate, is exactly whose or what experiences are likely to be accessible to us. We are thought to be able to empathise most easily with those closest to us and less well with experiences we do not understand. ${ }^{39} 40$ In this respect, in the context of abortion, Slote argues we are more likely to emphasise with a new born, rather than a fetus precisely because we cannot really have insight into the experience of a fetus. ${ }^{40}$

These points raise questions over the ease with which empathy can be promoted in the context of SUDs. Given that empathy is argued to be easiest for those you are closest to, it should follow that the significant others of people with SU problems find it easy to empathise with them. However, arguably the frustration family members may experience regarding another's substance use does not suggest closeness necessarily leads to empathy. This concern is exacerbated by the fact that empathy can be impaired among those with SUDs ${ }^{43}$; making the cultivation of empathy skills an important part of recovery. ${ }^{44}$ For significant others to empathise or 'get inside' the experience of those who can intermittently fail to show them the same respect, poses a significant moral challenge. This is important because families and significant others are increasingly seen to have an important role in recovery. ${ }^{45}$ That is, even though significant others have often been informed they need to wait for a substance user to want help, or 'hit rock bottom', they can use other, more positive strategies to help promote recovery. Like therapists, this includes significant others adopting an approach based on encouragement and empathy. But if empathising is actually ethically difficult for some significant others, it is doubtful how much responsibility they should be asked to assume. While training and support may help, the ethical requirements-relentless supererogation-may be too great to allow some to play a positive role. This being so, it is important that what is asked of people is ethically assessed on a case-by-case basis.

Finally, it is also necessary to consider the implications of this for therapist empathy. Given claims that empathy is easier for those 'closest' to the person, it might be thought to follow that therapists find it hard. However, the distance of therapists from the possible harms associated with a particular person's substance use, together with their professional, and sometimes personal, insights into SUDs suggests the benefits of familiarity can be achieved in a variety of ways. Yet the variation in therapist consultation styles and attitudes suggests reliance on empathy is far from unproblematic. In order to support such engagement in the context of SUDs, more dedicated ethical assessment is needed. As part of this work, it is important to lead debates away from a reliance on principles-like autonomy based on noninterference-which promote separateness over relatedness; and which lack the critical abilities to appraise behaviour-professional or client-that may be deleterious for health or strong relationships.

\section{The reach of relational autonomy}

Self-determination theory (SDT) has played an important role in driving a relational approach to substance use treatment. It is based on supporting three basic psychological needs-competence, relatedness and autonomy. ${ }^{46}$ Its exponents emphasise that autonomy does not have to be portrayed as independence. ${ }^{47}$ So it avoids the problems associated with a traditional bioethics approach, making SDT helpful in enriching debates around autonomy in the context of psychosocial treatments. In this respect, it shares much with feminist ethics and its relational concept of autonomy. That is, the conviction that people rarely become autonomous by being left to their own devices, but need support from others ${ }^{48}$; a view which is consistent and even imperative to a PCC approach. ${ }^{33}$ But as Ells et $a l^{35}$ argue, healthcare professionals can continue to operate with misconceived and outdated concepts of autonomy that prioritise noninterference to such an extent that clients are left isolated. As a result, the influence of relational autonomy is limited. Indeed, even those who argue for the importance of strong relationships use a traditional concept of autonomy when discussing professional ethics. ${ }^{45}$ This highlights that the relational standards at the heart of SDT are unlikely to be commonplace in treatment contexts for SUDs. For example, the ethics code of the NAADAC, the Association for Addiction Professionals in the USA defines autonomy simply as allowing 'others the freedom to choose their own destiny'. ${ }^{49}$

Apprehensions about the reach of relational autonomy are exacerbated by the fact that treatment and recovery do not take place in a social vacuum. It is not only health professional attitudes that matter for recovery efforts: Meier et al ${ }^{50}$ report, therapeutic relationships are aided by wider social networks. This makes it significant that in liberal democracies a narrow understanding of autonomy, linked to individual choice, tends to dominate public consciousness. As a result, a relational understanding of autonomy is not necessarily widespread among significant others or the general public. This hinders efforts to develop a prorecovery ethical culture around SUDs because the prominence of individual choice does not easily support a move away from blaming individuals for SUDs, or highlight the need for active, positive support from others. However, it is not only a failure to adopt a relational understanding of autonomy that impedes the development of a more beneficial approach to recovery, but the stigma that surrounds it. 


\section{CONFRONTING STIGMA}

At the heart of obstacles to creating an ethical culture that supports recovery is the stigmatisation of SUDs. Internationally, diagnostic tools for substance use issues vary, but both Diagnostic and Statistical Manual of Mental Disorders, Fifth Edition and International Classification of Diseases- 11 (ICD11) include characteristics such as a trong urge or impaired control over substance use; continuing to use a substance despite risks and mounting harm to self or others. ${ }^{12}$ Relapse is also a feature of substance use problems. These characteristics, and the negative stereotypes to which they give rise lead to SUDs being one of the most stigmatised health conditions. ${ }^{51}$ The stigmatisation of substance users is evident in the attitudes of both the general public and healthcare professionals. ${ }^{52}$ People with SUDs are often socially discredited or viewed as having 'spoiled identities. ${ }^{53}$ For a range of reasons, this plays a significant role in the availability of treatment for SUDs, its quality and accessibility. ${ }^{52}$ For example, external or structural stigma influences the level of financial support available for substance use problems and the way people are treated within health systems. ${ }^{54}$ Stigma perceived internally by the person with a substance use problem, can prevent treatment seeking and lead to feelings of exclusion. ${ }^{556}$ The role of stigma is particularly harmful because it undermines building the supportive relationships which are so critical for recovery.

\section{Stigma reduction}

In recent years, despite the harms associated with stigma, ${ }^{11}$ ethicists have been drawn into making and debating claims that stigma has a positive role in controlling behaviour-related chronic health conditions. ${ }^{5758}$ However, research identifies the importance of positive stigma reduction measures including: education, supporting healthcare providers to interact with people with stigmatised conditions, direct contact with stigmatised groups/individuals, empowering the stigmatised through a person-centred approach, questioning unconscious biases, advocacy and protest. ${ }^{52}$ Of importance to these different avenues to reducing stigma is, as Corrigan et al note in the context of mental health issues, the need to include strategies aimed at promoting 'affirming attitudes' around stigmatised conditions; this includes fostering hope and the belief that recovery is possible. ${ }^{59}$ In this respect, self-determination and individual choice are proposed as 'the crux' of building an affirmative recovery culture. ${ }^{59}$ The rationale for this is that self-determination affords a sense of empowerment. As we have seen, PCC also seeks to improve treatment quality and outcomes by giving greater importance to the voices of service users. However, I have argued that placing an unnuanced understanding of autonomy at the heart of PCC can undermine the creation of an ethical culture that supports recovery and actually perpetuate stigma. In the context of a condition marked by relapse, affirmation dependent on individual, client effort can quickly lead to claims people are irresponsible; and obscure the responsibilities others have to actively create supportive recovery environments.

\section{CONCLUSION}

For ethical and practical reasons, the choices of people with SUDs must be at the heart of efforts to develop a prorecovery ethical culture. However, a simple emphasis on self-determination and non-interference is insufficient. Without a stronger relational or social component, an individual focus can fuel a vicious circle which sees people with SUDs as responsible for their condition, blameworthy and the justifiable target of stigma. A reliance on self-determination also lacks the critical abilities to: support individuals to identify or establish their values-based choices; educate communities to respond constructively and proportionately to issues around SUDs; and move beyond a focus on individual clients to encourage the questioning of professional shortcomings. Finally, supporting the recovery process through individual choice or self-determination is challenging in social environments marked by an ambivalence, even hostility, towards those with SUDs. An ambivalence so great that many seeking to recover from alcohol or drug problems still opt to remain in 12-step programmes based on anonymity. Efforts to use individual affirmation to improve quality treatment and recovery chances must reconsider their ethical implications and the type of support that is actually required for the task at hand.

Acknowledgements I thank the peer reviewers of the paper for suggestions which have helped to improve the manuscript. I am grateful to Professor Jackie Tombs for conversations about the challenges of recovery, but ultimately, its attainability.

Contributors LW conceived, researched and wrote the paper.

Funding The authors have not declared a specific grant for this research from any funding agency in the public, commercial or not-for-profit sectors.

Competing interests None declared.

Patient consent for publication Not required.

Provenance and peer review Not commissioned; externally peer reviewed.

Data availability statement There are no data in this work.

Open access This is an open access article distributed in accordance with the Creative Commons Attribution Non Commercial (CC BY-NC 4.0) license, which permits others to distribute, remix, adapt, build upon this work non-commercially, and license their derivative works on different terms, provided the original work is properly cited, appropriate credit is given, any changes made indicated, and the use is non-commercial. See: http://creativecommons.org/licenses/by-nc/4.0/.

\section{ORCID iD}

Laura Williamson http://orcid.org/0000-0001-6461-2346

\section{REFERENCES}

1 American Psychiatric Association. Diagnostic and statistical manual of mental disorders (DSM-5). Arlington, VA: American Psychiatric Association, 2013.

2 World Health Organization. ICD-11 International classification of diseases 11th revision. Geneva: World Health Organization, 2020. https://icd.who.int/en

3 U.S. Department of Health and Human Services (HHS), Office of the Surgeon General. Facing Addiction in America: The Surgeon General's Report on Alcohol, Drugs, and Health. Washington, DC: HHS, 2016.

4 Wilson N, Kariisa M, Seth P, et al. Drug and Opioid-Involved Overdose Deaths - United States, 2017-2018. MMWR Morb Mortal Wkly Rep 2020;69(11):290-7.

5 Grant BF, Chou SP, Saha TD, et al. Prevalence of 12-Month Alcohol Use, High-Risk Drinking, and DSM-IV Alcohol Use Disorder in the United States, 2001-2002 to 2012 2013. JAMA Psychiatry 2017;74(9):911-23.

6 United Nations. Goal 3: ensure healthy lives and promote well-being for all at all ages. Available: https://www.un.org/sustainabledevelopment/development-agenda/ [Accessed 27 Jun 2020].

7 Dutra L, Stathopoulou G, Basden SL, et al. A meta-analytic review of psychosocial interventions for substance use disorders. Am J Psychiatry 2008;165(2):179-87.

8 Klein JW. Pharmacotherapy for substance use disorders. Med Clin North Am 2016;100(4):891-910.

9 Miller W, Andrews N, Wilbourne P, et al. A wealth of alternatives: Effective treatments for alcohol problems. In: Treating addictive behaviors. London and New York: Plenum Press, 1998: 203-10.

10 Carroll K. Treating drug dependence: Recent advances and old truths. In: Miller W, Heather N, eds. Treating addictive behaviors. London and New York: Plenum Press, 1998: 217-29

11 Institute of Medicine. Improving the quality of healthcare for mental and substanceuse conditions: the quality chasm series. Washington, DC: National Academies Press, 2006

12 Kelly JF, Bergman B, Hoeppner BB, et al. Prevalence and pathways of recovery from drug and alcohol problems in the United States population: implications for practice, research, and policy. Drug Alcohol Depend 2017;181:162-9.

13 Small D, Drucker E, Editorial for Harm Reduction Journal. Policy makers ignoring science and scientists ignoring policy: the medical ethical challenges of heroin treatment. Harm Reduct J 2006;3:16. 
14 Substance Abuse and Mental Health Service Administration. Working definition of recovery, 2012. Available: https://store.samhsa.gov/sites/default/files/d7/priv/pep12recdef.pdf [Accessed 27 Jun 2020].

15 Elster J, Skog 0-J. Getting hooked: rationality and addiction. Cambridge: Cambridge University Press, 1999

16 Carter A, Hall W, Illes J, eds. Addiction neuroethics: the ethics of addiction neuroscience research and treatment. Cambridge, MA: Academic Press, Elsevier, 2016.

17 Pickard H. Responsibility without blame for addiction. Neuroethics 2017:10(1):169-80.

18 Foddy B, Savulescu J. Addiction and autonomy: can addicted people consent to the prescription of their drug of addiction? Bioethics 2006:20(1):1-15.

19 Janssens MJPA, Van Rooij MFAM, ten Have HAMJ, et al. Pressure and coercion in the care for the addicted: ethical perspectives. J Med Ethics 2004;30(5):453-8.

20 Forrest AR. Ethical aspects of workplace urine screening for drug abuse. J Med Ethics 1997;23(1):12-17.

21 Hall W, Gartner C. Ethical and policy issues in using vaccines to treat and prevent cocaine and nicotine dependence. Curr Opin Psychiatry 2011:24(3):191-6.

22 Silkoff D, Guillemin M, Chenhall R, et al. What ethical resources are available to alcohol and other drug practitioners? A systematic review. Clin Ethics 2018:13(1):34-52.

23 Cohen E. 2006, Conceptualizing the professional relationship in drug user and misuser counseling. In: Kleinig J, Einstein S, eds. Ethical challenges for intervening in drug use: policy, research and treatment issues. Huntsville: Office of Criminal Justice: Huntsville, 2006: 367-81.

24 Levy N. The social: a missing term in the debate over addiction and voluntary control. Am J Bioeth 2007;7(1):35-6.

25 Institute of Medicine. Crossing the quality chasm: a new health system for the 21st century. Washington, DC: National Academy Press, 2001

26 Florin D, Dixon J. Public involvement in health care. BMJ 2004;328(7432):159-61.

27 Norcross JC, Wampold BE. Evidence-based therapy relationships: research conclusions and clinical practices. Psychotherapy 2011;48(1):98-102.

28 Najavits LM, Weiss RD. Variations in therapist effectiveness in the treatment of patients with substance use disorders: an empirical review. Addiction 1994;89(6):679-88

29 Rogers C. A way of being Boston. MA: Houghton Mifflin, 1980

30 Volpicelli J, Szalavitz M. Recovery options: the complete guide. Hoboken, NJ: Wiley, 2000.

31 Valle SK. Interpersonal functioning of alcoholism counselors and treatment outcome. J Stud Alcohol 1981:42(9):783-90.

32 Bradley KA, Kivlahan DR. Bringing patient-centered care to patients with alcohol use disorders. JAMA 2014:311(18):1861-2.

33 Lindsay B. Atheist nurse wins fight to end mandatory 12-step addiction treatment for health staff in Vancouver, 2016. Available: https://www.cbc.ca/news/canada/ british-columbia/bc-byron-wood-nurse-12-step-religious-discrimination-settlement-1. 5391650 [Accessed 27 Jun 2020].

34 White W. Toward a philosophy of choice: a new era of addiction treatment. Counselor 2008;9(1):38-43.

35 Ells C, Hunt MR, Chambers-Evans J. Relational autonomy as an essential component of patient-centered care. IJFAB: Int J Feminist Approach Bioethic 2011:4(2):79-101.

36 Williamson L. Patient and citizen participation in health: the need for improved ethica support. Am J Bioeth 2014;14(6):4-16.
37 Gessert C. The problem with autonomy: an overemphasis on patient autonomy results in patients feeling abandoned and physicians feeling frustrated. Minnesota Med 2008;91(4):40-2

38 Schwartz RP, Kelly SM, Mitchell SG, et al. Patient-Centered methadone treatment: a randomized clinical trial. Addiction 2017:112(3):454-64.

39 Coplan AMY. Will the real empathy please stand up? A case for a narrow conceptualization. South J Philos 2011:49(S1):40-65.

40 Slote M. The ethics of care and empathy. London and New York: Routledge, 2007.

41 van Dijke J, van Nistelrooij I, Bos P, et al. Towards a relational conceptualization of empathy. Nurs Philos 2020;21(3).

42 Graber MA, Ely JW. Empathy is a poor Foundation on which to base legislative medical policy. Bioethics 2018;32(7):402-4.

43 Martinotti G, Di Nicola M, Tedeschi D, et al. Empathy ability is impaired in alcoholdependent patients. Am J Addict 2009;18(2):157-61.

44 Ferrari V, Smeraldi E, Bottero G, et al. Addiction and empathy: a preliminary analysis. Neurol Sci 2014;35(6):855-9.

45 Miller W, Forcehimes A, Zweben A. Treating addiction: a guide for professionals. New York and London: Guildford Press, 2019.

46 Ryan R, Deci E. 2002 Overview of self-determination theory: an organismic dialect perspective. In: Deci $\mathrm{E}$, Ryan R, eds. Handbook of self-determination theory. Rochester, New York: University of Rochester Press, 2002: 3-33.

47 Ryan RM. Psychological needs and the facilitation of integrative processes. J Pers 1995;63(3):397-427.

48 Stoljar N. Informed consent and relational conceptions of autonomy. J Med Philos 2011;36(4):375-84.

49 NAADAC: association for addiction professionals, NCC AP: the National certification Commission for addiction professionals, code of ethics Approved 10.09.2106. Available: https://www.naadac.org/assets/2416/naadac-nccap-code-of-ethics11-0416.pdf [Accessed 27 Jun 2020].

50 Meier PS, Barrowclough C, Donmall MC. The role of the therapeutic alliance in the treatment of substance misuse: a critical review of the literature. Addiction 2005; 100(3):304-16.

51 Link BG, Phelan JC, Bresnahan M, et al. Public conceptions of mental illness: labels, causes, dangerousness, and social distance. Am J Public Health 1999:89(9):1328-33.

52 Knaak S, Mantler E, Szeto A. Mental illness-related stigma in healthcare: barriers to access and care and evidence-based solutions. Healthc Manage Forum 2017:30(2):111-6.

53 Goffman E. Stigma: notes on the management of spoiled identity. Englewood cliffs, NJ: Prentice-Hall, 1963.

54 Olsen JA, Richardson J, Dolan P, et al. The moral relevance of personal characteristics in setting health care priorities. Soc Sci Med 2003;57(7):1163-72.

55 Schomerus G, Corrigan PW, Klauer T, et al. Self-stigma in alcohol dependence: consequences for drinking-refusal self-efficacy. Drug Alcohol Depend 2011;114(1):12-17.

56 Yang L, Wong L, Grivel M, et al. Stigma and substance use disorders: an international phenomenon. Curr Opinion Psych 2017;30(5):378-88.

57 Bayer R. Stigma and the ethics of public health: not can we but should we. Soc Sci Med 2008:67(3):463-72.

58 Callahan D. Obesity: chasing an elusive epidemic. Hastings Cent Rep 2013:43(1):34-40.

59 Corrigan PW, Angell B, Davidson L, et al. From adherence to self-determination: evolution of a treatment paradigm for people with serious mental illnesses. Psychiatr Serv 2012:63(2):169-73. 